\title{
Engeler's scientific work
}

\author{
E. SPECKER†
}

Zurich

Received 5 December 1991

Erwin Engeler was born in Schaffhausen on 13 February 1930 a citizen of Wagenhausen (Thurgovia). He attended school in Diessenhofen (TG) and Schaffhausen and looks back on his various schools warmly as having provided a conducive environment. His schoolwork also left him time for other activities. For one, he pursued a career as a boy scout which he crowned by attaining the position of Rover Commissary of all Thurgovia. For another, he was an avid client of the municipal library of Schaffhausen. One book which he found there was Hilbert-Bernays' 'Grundlagen der Mathematik'. One wonders what Erwin would have said if a soothsayer had told him that the author's own copy would one day be passed on to him by the late Bernays' family.

He graduated from school in 1950 and entered the ETH (The Federal Institute of Technology) in Zurich to study mathematics. He was awarded a diploma in 1955 and a doctor's degree in 1958, both accomplished under the supervision of Bernays. While preparing his doctoral thesis, he worked for an insurance company. In 1956 Erwin and Margaret Knecht were married; both their lives from that point on cannot be conceived without each other.

In 1958 they left Zurich for Minneapolis, where Erwin occupied at first an assistant professorship from which he was in due time promoted. He remained in Minneapolis until 1972 with the exception of two years' leave in 1962/1964, which he spent at the University of California at Berkeley, at the IBM research laboratory in Rüschlikon (Zurich) and at the ETH. In 1972 he returned to work in Zurich, where he was offered a professorship for Logic and Computer Science. As in Minnesota, Engeler's activities at the ETH span a wide range. He played an essential role in the Center for Interactive Computing and has been for many years a member of the Swiss National Research Council.

The same diversity characterizes his scientific work. If we have to formulate a single heading under which to put his work, the following seems to fit best: Structure and Language. There seems to be a kind of complementary relation between these two poles, a relation which is far from understood and can (at the moment) be elucidated only by studying particular aspects. To give an idea of Engeler's contribution to this fundamental problem, a structured list of his papers is adjoined and six of these are reviewed in some detail. A selection of papers will be published under the title 'Algorithmic Properties of Structures' by the World Scientific Publishing Corporation in May 1992.

The first of the papers to be reviewed here (1959) [2] is related to Cantor's theorem

$\dagger$ G. Weibel and St. Collart are thanked for their help. 
according to which every denumerable ordered set satisfying the same sentences of firstorder logic as the rationals is isomorphic to the rationals (1959) [2]. Engeler realized-presumably by analysing Cantor's back-and-forth argument - that the 'reason' for this is the following: for every $m$, there is a system of finitely many $m$-tuples $\left(x_{1}, \ldots, x_{m}\right)$ of rationals such that every $m$-tuple of rationals satisfies the same sentences of first-order logic as some member of the system. (For $m=2,(0,0),(0,1),(1,0)$ constitute such a system.) This result has come to be known as the theorem of Engeler-RyllNardzewski-Svenonius.

As is well known, first-order logic is inadequate to express important mathematical properties. The axiom of Archimedes is an example of such a property.

However small a positive step $s$, any point $p$ can be surpassed with sufficiently many iterations of this step $s$.

This property can also be expressed by the following formula of infinite length:

$$
p<s \vee p<(s+s) \vee p<(s+s+s) \vee \ldots \vee p<(2+s+\ldots+s) \vee \ldots
$$

If first-order logic is generalized to allow infinite disjunctions

$$
\left(\Phi_{1} \vee \Phi_{2} \vee \ldots\right)
$$

the question then arises: under what condition is such a disjunction equivalent to a proper first-order sentence? Engeler's answer is as follows: if $\neg\left(\Phi_{1} \vee \Phi_{2} \vee \ldots\right)$ is equivalent to an infinite disjunction

$$
\left(\Psi_{1} \vee \Psi_{2} \vee \ldots\right)
$$

of first-order formulae $\Psi_{i}$, it is equivalent to a first-order formula (equivalence being defined by validity in the models of a first-order theory) (1961) [4].

During the years 1964-1967, Engeler presented a very important generalization of firstorder logic in lectures given at the University of Minnesota and the ETH in Zürich. His ideas are contained in the book 'Formal Languages' (1967) [14].

Consider the following program $\Pi_{0}$ :

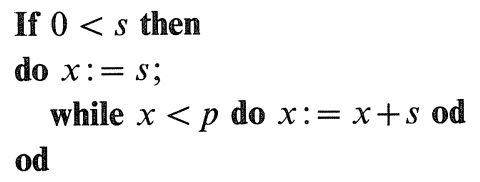

Clearly this program terminates in an ordered group $G$ for all values of $s$ and $p$ if and only if $G$ satisfies the Archimedean axiom. Defining $S \models \Pi$ as ' For all instances $\Pi^{\prime}$ of $\Pi$ the program $\Pi^{\prime}$ terminates', the following is a form of the axiom of Archimedes

$$
G \models \Pi_{0} .
$$

The relation $\vDash$ being defined, many notations of classical logic immediately generalize to 'dynamic logic', e.g.

two structures $S, S^{\prime}$ are algorithmically equivalent if and only if for all programs $\Pi$

$$
S \models \Pi \Leftrightarrow S^{\prime} \vDash \Pi \text {. }
$$


The power of this approach is exemplified by showing how these new definitions allow the theory of constructions to be treated in elementary geometry. Problem:

find a construction by ruler and compass of an angle of 1 degree.

As is well known, such a construction is impossible. In order to give a proof meeting contemporary standards of rigour, constructions are defined in the natural way as programs. $\Pi_{k}$ is a program which multiplies a given angle by 360 and terminates if and only if the resulting angle is full. The problem of the existence of a construction of an angle of $1^{\circ}$ then reduces to the question: does there exist a program $\Pi_{1}$ such that

$$
E G \models \Pi_{1} ; \Pi_{k} \text { ? }
$$

( $E G$ is the structure of Euclidean geometry based on the real field.) The proof that there is no construction of an angle of degree 1 then proceeds in the following two steps:

(1) A geometric structure $A G$ is defined such that $A G$ is algorithmically equivalent to $E G$.

(2) In $A G$ there does not exist an angle of 1 degree.

In the paper 'An Algorithmic Model of Strict Finitism' (1978) [34] an attempt is made to give a definition of strict finitism in the frame of classical mathematics. The fundamental idea is to introduce a series of mathematicians, waxing in competence and in patience, and to examine where they are in final agreement. Engeler has so far been followed by a $\mathrm{PhD}$ student (E. Welti) who continued the original 12 page paper in a treatise of some 625 pages.

'Generalized Galois Theory and its applications to Complexity' (1981) [30] is perhaps the paper most typical of Engeler's scientific activity. Starting from one of the most central themes of mathematics (roots of polynomials), a general theory for a central problem of computer science is developed. We state both of these problems in their simplest form:

(1) Under what conditions can the roots of a polynomial - such as $x^{5}+x=3$ - be expressed by radicals (i.e. functions $\sqrt{ }, \sqrt[3]{ }, \ldots)$ ?

(2) Given a program $\Pi$, and programs $\Pi_{1}, \Pi_{2}, \ldots$, can $\Pi$ be represented as

$$
\Pi=\Pi_{i_{1}} ; \Pi_{i_{2}} ; \ldots ; \Pi_{i_{n}} ?
$$

It is obvious how a positive answer will (in general) be given : by exhibiting the sequence $\Pi_{i_{j}}$. But how can we show that the answer is negative? Engeler shows how a generalization of the group theoretic methods of Galois permits such proofs for a wide range of structures and problems.

In the last paper under review (Representation of Varieties in Combinatory Algebras) (1988) [46], a new type of structure is defined, appropriately called 'Engeler Universes'. These structures provide models for combinatorial logic and the $\lambda$-calculus. Combinatorial logic goes back to Moses Schönfinkel, a friend of Bernays', in whose seminars it was a recurrent topic. Its original object was to furnish a foundation for mathematics and the question of models therefore did not arise. The situation changed when the importance of $\lambda$-calculus in the context of programming languages was realized. Models were then studied by D. Scott and G. D. Plotkin. The extreme simplicity of the models introduced by Engeler makes it possible to present his definition in detail. 
Given a set $A$, let $G(A)$ be the smallest set containing $A$ as a subset and closed under the adjunction of $(\alpha \rightarrow b)$, where $\alpha$ is a finite subset of $G(A)$ and $b$ an element of $G(A)$.

On the power set $B$ of $G(A)$ a binary operation $\circ$ is defined as follows.

For $M, N \in B, M \circ N$ is the set of elements $b$ for which there exists $\alpha, \alpha \subseteq N$, such that $(\alpha \rightarrow b) \in M$.

The resulting structure $(B, \circ)$ turns out to be a combinatory algebra, i.e. for every term $\Phi\left(x_{1}, \ldots, x_{n}\right)$ defined with the help of elements of $B$ and variables $x_{1}, \ldots, x_{n}$, there exists an element $f, f \in B$, such that for all $a_{1}, \ldots, a_{n}$ in $B$

$$
\Phi\left(a_{1}, \ldots, a_{n}\right)=\left(\ldots\left(\left(f \circ a_{1}\right) \circ a_{2}\right) \ldots a_{n}\right) .
$$

In short: every definable function in $B$ is represented by an element of $B$-whatever can be imagined is already achieved.

The author is aware that he has not succeeded in doing full justice to Engeler's work. However, he is convinced that he is more successful in his role as a soothsayer: some day he will be followed by a reviewer more adept in competence and in patience.

\section{Publications 1958-1991}

1. Classical model theory and infinitary languages

[1] Untersuchungen zur Modelltheorie. Diss. ETH (1958).

[2] Äquivalenzklassen von n-Tupeln. Z. Math. Logik Grundl. Math. 5 (1959) 340-345.

[3] Eine Konstruktion von Modellerweiterungen. Z. Math. Logik Grundl. Math. 5 (1959) 126-131.

[4] Unendliche Formeln in der Modelltheorie. Z. Math. Logik Grundl. Math. 7 (1961) 154-160.

[5] Zur Beweistheorie von Sprachen mit unendlich langen Formeln. Z. Math. Logik Grundl. Math. 7 (1961) 213-218.

[6] A reduction principle for infinite formulas. Mathem. Annalen 151 (1963) 296-303.

\section{Categories in model theory}

[7] Models with prescribed second-order properties. J. Symbolic Logic 27 (1962) 476.

[8] Combinatorial theorems for the construction of models. In: The Theory of Models. Proc. 1963 Int. Symp., eds J. W. Addison et al. (North-Holland: Amsterdam, 1965) pp. 77-88.

[9] Categories of mapping filters. In: Conf. Categorical Algebra, ed. S. Eilenberg et al. (Springer: New York, 1966) pp. 247-253.

[10] On structures defined by mapping filters. Math. Annalen 167 (1966) 105-112.

[11] On the structure of elementary maps. Z. Math. Logik u. Grundl. d. Math. 13 (1967) 323-328.

[12] (with H. Röhrl) On the problem of foundations of category theory. Dialectica 23 (1969) 58-66. 
3. Algorithmic properties of structures and logics of programs

[13] Algorithmic properties of structures. Math. Systems Theory 1 (1967) 183-195.

[14] *Formal Languages: Automata and Structures. (Markham: Chicago, 1968).

[15] Remarks on the theory of geometrical constructions. In: Syntax \& Semantics of Inf. Lang., ed. J. Barwise, Springer Lecture Notes in Mathematics 72 (Springer: New York, 1968) pp. 64-76.

[16] Proof theory and the accuracy of computations. In: Symp. Automatic Demonstr., eds M. Laudet et al., Springer Lecture Notes in Mathematics 125 (Springer: New York 1970) pp. 62-72.

[17] Algorithmic approximations. J. Comput. Systems Sci. 5 (1971) 67-82.

[18] Structure and meaning of elementary programs. Symp. Sem. of Alg. Lang. Springer Lectures Notes in Mathematics 188 (Springer: New York, 1971) pp. 89-101.

[19] *(ed.) Symposium on Semantics of Algor. Languages. Springer Lecture Notes in Mathematics 188 (Springer: New York, 1971).

[20] *Introduction to the Theory of Computation. (Academic Press: New York, 1973). (Transl. into Japanese 1975).

[21] The logic of 'can do', Logic of Programming. In: Int. Symp. on Theoretical Computer Science, eds A. Ershov et al., Springer Lecture Notes in Computer Science 5 (1974) pp. 17-28.

[22] Algorithmic logic. Math. Center Tracts 63 (1975) 57-85.

[23] *(ed.) Logic of Programs. Springer Lecture Notes in Computer Science 125 (Springer: New York, 1981).

[24] Logic in Computer Science. In: Information Processing 86, ed. H. J. Kugler (NorthHolland, Amsterdam, 1986), 393.

4. Galois-connections and the structure of programs

[25] On the structure of algorithmic problems. GI Fachtagung über Automatentheorie und formale Sprachen. Springer Lecture Notes in Computer Science 2 (Springer: New York, 1973) pp. 2-15.

[26] Towards a Galois theory of algorithmic problems. Math. Found. of Comp. Sc., eds J. Becvár et al., (Slovac Academy of Science: 1973) pp. 51-55.

[27] On the solvability of algorithmic problems. Logic Colloquium 73 eds, H. E. Rose et al. (North-Holland, Amsterdam, 1975), pp. 231-251.

[28] Lower bounds by Galois theory. Asterisque 38 (1976) 45-52.

[29] Structural relations between programs and problems. Int. Congr. Logic Math. \& Phil. Sci. London Ont. 1975, Logic, Found. of Math. and Computability, eds Butts et al. (Reidel: Dordrecht, 1977) pp. 267-280.

[30] Generalized Galois theory and its application to complexity. Theoret. Comput. Sci. 13 (1981) 271-293.

5. Foundations and philosophy of science

[31] Geometry and language. Dialectica 24 (1970) 77-85. 
[32] *Metamathematik der Elementarmathematik. (Springer: New York, 1983).

[32a] (Transl. into Russian 1987, Transl. into English and into Chinese in preparation.)

[33] Zum logischen Werk von Paul Bernays. Dialectica 32 (1978) 191-200.

[34] An algorithmic model of strict finitism. Colloq. math. J. Bolyai (1978) 345-357.

[35] Die Skepsis der Mathematiker gegenüber der Grundlagenrhetorik. In: Wozu Wissenschaftsphilosophie eds P. Hoyningen-Huene et al. (de Gruyter, 1988) pp. 310-313.

[36] Zur wissenschaftstheoretischen Bedeutung der kombinatorischen Algebra. In: Jahrbuch der Kurt Gödel Gesellschaft (Wien 1990).

[37] The technical problem of 'full abstractness' as a model for an issue in reductionism. In: The Problem of Symposium on Reductionism in Science, ed. E. Agazzi (Kluwer, 1991) pp. 91-99.

\section{Combinatory algebra as a functional structure for mathematics}

[38] A new type of models of computation. Math. Found. Comp. Sci., ed. J. Gruska, Springer Lecture Notes in Computer Science 53 (Springer: New York, 1977) pp. $52-58$.

[39] Formal models of computation in which data are processes: theory and applications. Int. Conf. Math. Stud. Info. Proc. Kyoto (1978) pp. 541-558.

[40] Algebras and combinators. Algebra Universals 13 (1981) 389-392.

[41] Equations in combinatory algebras. In: Logics of Programs, eds E. Clarke et al., Springer Lecture Notes in Computer Science 164 (Springer: New York, 1984) pp. 193-205.

[42] Modelling of cooperative processes. In: Comput. Theory and Logic, ed. E. Börger, Springer Lecture Notes in Computer Science 270 (Springer: New York, 1987) pp. 143-153.

[43] Cumulative type logic programs and modelling. J. Symbolic Logic 52 (1987) 1059.

[44] Cumulative logic programs and modelling. In: Logic Colloquium 1986, eds F. R. Drake et al. (North-Holland, Amsterdam, 1988) pp. 83-93.

[45] Modelling interacting intelligent systems in the legal context. Neue Methoden im Recht 5 (1988).

[46] Representation of varieties in combinatory algebras. Neue Methoden im Recht 25 (1988) 85-95.

[47] A new discipline of modelling. Report ETH Zürich (1988).

[48] Combinatory differential fields. Theore. Comput. Sci. 72 (1990) 119-131.

\section{Miscellaneous publications}

[49] Analogie-Rechengerät ARZ. Anleitung, Güttinger Niederteufen (1956).

[50] Über die Monte-Carlo Methode. Mitt. Schweiz. Vers.-math. 58 (1958) 67-76.

[51] Lineares Programmieren. Mitteilungsblatt Nr. 4 (Güttinger, 1958). 
[52] (with A. P. Speiser). Zur Analogie zwischen einer elektronischen Rechenmaschine und dem Gehirn. Vierteljahresschr. d. Natf. Gesellsch. in Zürich 109 (1958) 81-84.

[53] Über elektr. Analogierechenanlagen für chem. \& biol. Vorgänge. Jahrbuch Chem. Industrie 1958/59, 92-95.

[54] (with R. Mäder). Scientific Computation: The integration of symbolic, numeric and graphic computation. In: Eurocal '85, ed. B. Buchberger, Springer Lecture Notes in Computer Science 203 (Springer: New York, 1985) pp. 185-200.

[55] Goals and design consideration for a mathematical laboratory. SIGSAM Bulletin 21 (1987) 7-8.

[56] Grenzen-des Computers? (= Introduction to) Grenzen des Computers, ed. C. Schnell (Sauer: 1991). 